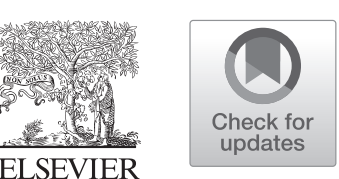

\title{
Nursing Care of the Patient With Neurogenic Bladder After Kidney Transplantation: A Case Report
}

\author{
Zeliha Ozdemir Koken ${ }^{\mathrm{a}, *}$, Rana Elcin Sezer ${ }^{\mathrm{a}}$, and Kubra Tosun ${ }^{\mathrm{b}}$ \\ asurgical Nursing Department, Hacettepe University Faculty of Nursing, Ankara, Turkey; and bepartment of Urology, Hacettepe \\ University Adult Hospital, Ankara, Turkey
}

\begin{abstract}
Objective. Urologic complications are among the most common complications after kidney transplantation. These complications are urinary retention, hematuria, hemorrhage, urinary leakage, vesicoureteral reflux, pyelonephritis, and nephrolithiasis. Although neurogenic bladder is one of the indications for kidney transplantation, it is not considered in the literature to be an expected complication after transplantation. In this case, we discuss the nursing care of a patient who underwent kidney transplantation from a living donor and developed neurogenic bladder.

Case report. A 60-year-old woman underwent kidney transplantation from a living donor, and neurogenic bladder developed in the patient 1 year after kidney transplantation. Clear intermittent catheterization treatment was administered for the kidney transplant recipient with neurogenic bladder. Clear intermittent catheterization treatment was stopped in the patient who had frequent urinary tract infections and, alternatively, sacral neuromodulation treatment was administered to the patient.

Conclusions. The nursing care of a patient with neurogenic bladder after kidney transplantation aims to prevent excessive bladder distension, infection, stone formation, vesicoureteral reflux, renal failure, urinary tract damage, and incontinence, and to ensure regular and complete discharge of the bladder. The most common treatment modalities for these objectives are permanent or intermittent catheterization, sacral neuromodulation, and medical therapy. In the care of the patient with neurogenic bladder after kidney transplantation, nurses should provide appropriate care related to treatment options and bladder training, plan urination schedules of the patient, and monitor for possible complications.
\end{abstract}

$\mathbf{T}$ HE MOST common post-transplant urologic complications are urinary retention, hematuria, hemorrhage, urinary leakage, vesicoureteral reflux, pyelonephritis, and nephrolithiasis [1,2]. Although neurogenic bladder is one of the indications for kidney transplantation, it is not considered in the literature to be an expected post-transplant complication $[2,3]$. In this study, we discuss the nursing care of a patient with polycystic kidney disease who underwent kidney transplantation from a living donor and subsequently developed a neurogenic bladder.

\section{CASE PRESENTATION}

A 60 -year-old female patient with polycystic kidney disease who was monitored regularly in 1996 underwent kidney transplantation from her sister in 2012. After the transplantation, she was diagnosed with neurogenic bladder in 2013 because of a postvoid residual urine volume of approximately 200.0 to $300.0 \mathrm{~mL}$; as a result, she underwent clean intermittent catheterization (CIC). She had a urinary tract infection due to $\mathrm{CIC}$ and received antibiotic treatment. Because of frequent urinary tract infections, the CIC was stopped, and she underwent sacral neuromodulation in 2013. She underwent CIC (four times per day) in March 2018 because of postvoid

*Address correspondence to Zeliha Ozdemir Koken, PhD, Hacettepe University Faculty of Nursing, Adnan Saygun Street, D-Block, 1st Floor, 06100-Altindag, Ankara, Turkey. Tel.: +90 31230515 80; Fax: +90 31231270 85. E-mail: ozdemir.z@ hotmail.com

$0041-1345 / 19$

https://doi.org/10.1016/j.transproceed.2019.01.187 
residual urine in routine control. She had urinary tract infection due to CIC and was admitted to the clinic and underwent antibiotic treatment. After the antibiotic treatment had been completed, she was discharged and continued on CIC. She was infected with Escherichia coli and underwent antibiotic treatment again in August 2018.

\section{DISCUSSION}

Neurogenic bladder is a dysfunction resulting from a defect in nerve conduction that innervates the bladder [1]. Stroke, Parkinson disease, multiple sclerosis, spinal cord injury, and central or peripheral nervous system damage can cause neurogenic bladder. Neurogenic bladder results in urinary retention and voiding dysfunction, urinary tract infections, bladder neck obstruction, and renal failure [1,4,5]. Renal failure is 8 times more prevalent in patients with neurogenic bladder than in patients without neurogenic bladder [6]. Proper treatment and care is, therefore, extremely important to prevent the risk of renal failure, to maintain graft function, and to extend graft life, as in our patient who developed posttransplant neurogenic bladder [3]. The choice of neurogenic bladder therapy depends on the general condition of the patient, the cause of the neurogenic bladder, and the patient's preference and expectations. The aim of the treatment is to prevent excessive bladder distension, infection, stone formation, vesicoureteral reflux, and renal failure and to ensure regular and complete discharge of the urinary bladder. Neurogenic bladder has many treatment options including surgery, medical treatment, and catheterization. Appropriate treatment selection and regular follow-up and care prevent neurogenic bladder complications [5,7]. Anticholinergic, antimuscarinic, and antidiuretic drugs are used in medical treatment [5,7-9]. Surgery is the treatment of choice when medical treatment is not effective. Urinary diversion, bladder augmentation, and sacral neuromodulation are used in surgical treatment [7]. In our case, the patient underwent medical treatment and CIC. In catheterization, the patient's bladder is drained of urine-usually every 4 to 6 hours-by catheterization performed by a caregiver using a sterile technique in the hospital. When the patient is discharged, the bladder is emptied through CIC at home [5,7]. CIC is a safe and effective method for emptying the bladder by using a clean catheter in cases of neurogenic bladder or other bladder-voiding problems. Although CIC is the method of choice in treatment, $80 \%$ of patients have CIC-associated urinary tract infection [10]. This situation should be taken into account, especially in the care of the kidney transplant recipient who is taking immunosuppressive medications. In our case, the kidney transplant recipient who underwent $\mathrm{CIC}$ treatment had frequent urinary tract infections. Nurses and other health professionals should be trained about catheter management, and indications and application times for catheter administration should be standardized in order to prevent urinary tract infections in patients diagnosed with neurogenic bladder. In addition, hands should be washed with soapy water prior to CIC, the genital area should be cleaned once a day with water and soap, and it should be cleaned with only water during the day. In CIC, nurses should plan hands-on training according to their patients' age, educational status, and sociocultural characteristics. During training, patients should be informed about the anatomy of the urinary system, important aseptic techniques for before and after the application, how to insert and remove the catheter, and catheter care [10]. The kidney transplant recipient who had frequent urinary tract infections due to CIC while experiencing very little benefit from the treatment was ultimately treated with sacral neuromodulation. Sacral neuromodulation is simple, safe, and reversible. In this treatment, the bladder is stimulated by an electrode placed in the sacrum allowing the patient to void. The responsibilities of nurses in sacral neuromodulation are monitoring fluid intake and urinary output, recording the frequency of urination, and recording the amount of urine produced [7].

\section{CONCLUSIONS}

In the care of patients with neurogenic bladder, nurses should offer bladder training, plan urination schedules for their patients, provide care appropriate to treatment options, monitor possible complications and supervise pelvic floor exercises [7]. Nurses should be familiar with new treatment options for neurogenic bladder management, and plan care in line with current information $[7,8]$. Nurses play a more significant role in preventing the development of urinary tract infections and renal failure in kidney transplant recipients who develop neurogenic bladder after transplantation.

\section{REFERENCES}

[1] Ginsberg D. The epidemiology and pathophysiology of neurogenic bladder. Am J Manag Care 2013;19:s191-6.

[2] Di Carlo HN, Darras FS. Urologic considerations and complications in kidney transplant recipients. Adv Chronic Kidney Dis 2015;22:306-11.

[3] Whittaker C, Dunsmore V, Murphy F, Rolfe C, Trevitt R. Long-term care and nursing management of a patient who is the recipient of a renal transplant. J Ren Care 2012;38:233-40.

[4] Przydacz M, Denys P, Corcos J. What do we know about neurogenic bladder prevalence and management in developing countries and emerging regions of the world? Ann Phys Rehabil Med 2017;60:341-6.

[5] Goldmark E, Niver B, Ginsberg DA. Neurogenic bladder: from diagnosis to management. Curr Urol Rep 2014;15:448.

[6] Lawrenson R, Wyndaele JJ, Vlachonikolis I, Farmer C, Glickman S. Renal failure in patients with neurogenic lower urinary tract dysfunction. Neuroepidemiology 2001;20:138-43.

[7] Pellatt GC, Geddis T. Neurogenic continence. Part 2: neurogenic bladder management. Br J Nurs 2008;17:904-13.

[8] Woodward S. Current management of neurogenic bladder in patients with MS. Br J Nurs 2004;13:362-70.

[9] Sripathi V, Mitra A. Management of neurogenic bladder. Indian J Pediatr 2017;84:545-54.

[10] Willson M, Wilde M, Webb M-L, Thompson D, Parker D, Harwood J, et al. Nursing interventions to reduce the risk of catheter-associated urinary tract infection: part 2 staff education, monitoring and care techniques. J Wound Ostomy Continence Nurs 2009;36:137-54. 